\title{
Anterior Chamber Non-Hodgkin Lymphoma of the Iris Masquerading as Uveitis-Glaucoma-Hyphema Syndrome
}

\author{
Angela C. Gauthier ${ }^{\mathrm{a}}$ Alexander Nguyen ${ }^{\mathrm{a}}$ William R. Munday ${ }^{\mathrm{b}}$ Mina L. Xu ${ }^{\mathrm{b}}$ \\ Miguel A. Materin ${ }^{a, c}$ \\ Departments of a Ophthalmology and Visual Science and ${ }^{b}$ Pathology, Yale School of Medicine, and \\ 'Ophthalmic Oncology, Smilow Cancer Hospital, New Haven, Conn., USA
}

\section{Key Words}

Non-Hodgkin lymphoma · Iris · Hyphema · Uveitis · Mantle cell lymphoma

\begin{abstract}
Purpose: To report a case of iris non-Hodgkin lymphoma initially thought to be uveitis-glaucoma-hyphema (UGH) syndrome. Methods: We reviewed the clinical, radiographic, and histopathologic findings in a patient with recurrent hyphemas and increased ocular pressure who eventually was found to have a rapidly growing iris mass. Results: An 89-year-old man with a history of cataract extraction and mantle cell lymphoma developed recurrent hyphema, which was subsequently revealed to be due to an iris mass. A biopsy revealed non-Hodgkin lymphoma that could not be formally subclassified but was suspicious for mantle cell lymphoma. The tumor showed a partial response to ibrutinib. Conclusion: Iris lymphoma can masquerade as a cause of recurrent hyphema after cataract extraction. Ophthalmologists should be aware of this presentation, especially in patients with a history of lymphoma.
\end{abstract}

(c) 2016 S. Karger AG, Basel
C 2016 S. Karger AG, Basel

2296-4681/16/0024-0230\$39.50/0

\section{Introduction}

Non-Hodgkin lymphoma (NHL) is a group of cancers that derive from lymphocytes, often localizing to lymph nodes. It makes up approximately $5.1 \%$ of all cancer cases, and it causes $2.7 \%$ of all cancer deaths [1]. If it involves the eye, NHL usually localizes to eye adnexal structures or the posterior eye $[2,3]$. Only rarely does it involve the iris [4-6]. Iris lymphomas associated with systemic lymphoma tend to be very aggressive, and they are often initially misdiagnosed as anterior uveitis [6]. Iris lymphoma may also appear to be uveitis-glaucoma-hyphema (UGH) syndrome, especially in patients who have had previous cataract extraction and subsequent periodic hyphemas [7]. Herein we describe a patient with recurrent iris NHL masquerading as UGH syndrome.

\section{Case Report}

An 89-year-old white man was referred to ophthalmic oncology for evaluation of an anterior segment mass involving the iris in the left eye. The patient had a history of cataract extraction and intraocular lens implantation in the left eye approximately 5 years prior. For over a year prior to referral, the patient had recurring

\section{KARGER}

E-Mail karger@karger.com

www.karger.com/oop
Alexander T. Nguyen, MD

Department of Ophthalmology and Visual Science

Yale School of Medicine, 40 Temple Street, Suite 3B

New Haven, CT 06510 (USA)

E-Mail alexander.nguyen@yale.edu 


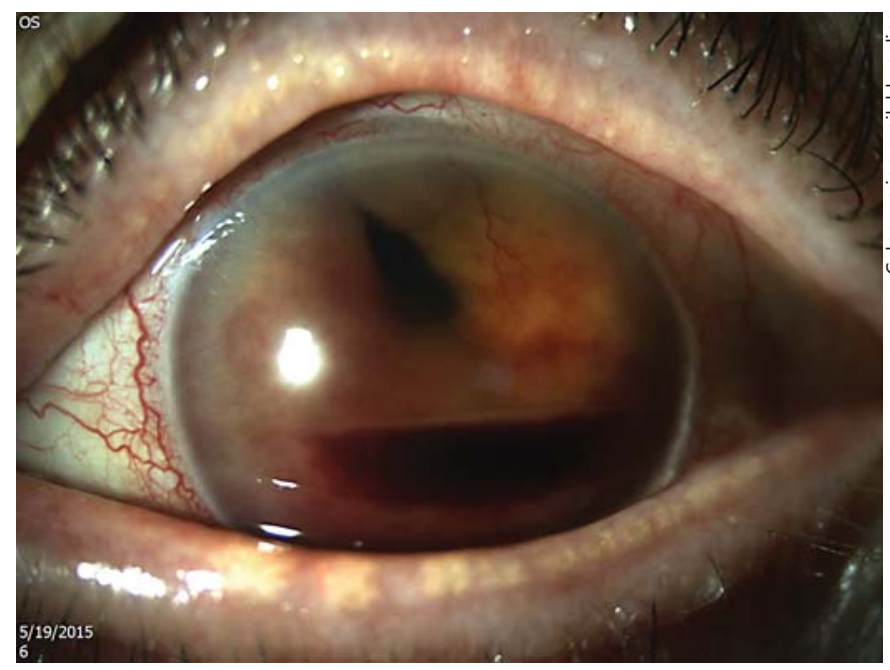

Fig. 1. Photograph of the left eye at presentation showing dilated episcleral vessels, anterior chamber hyphema, and circumferential engorgement of the iris.

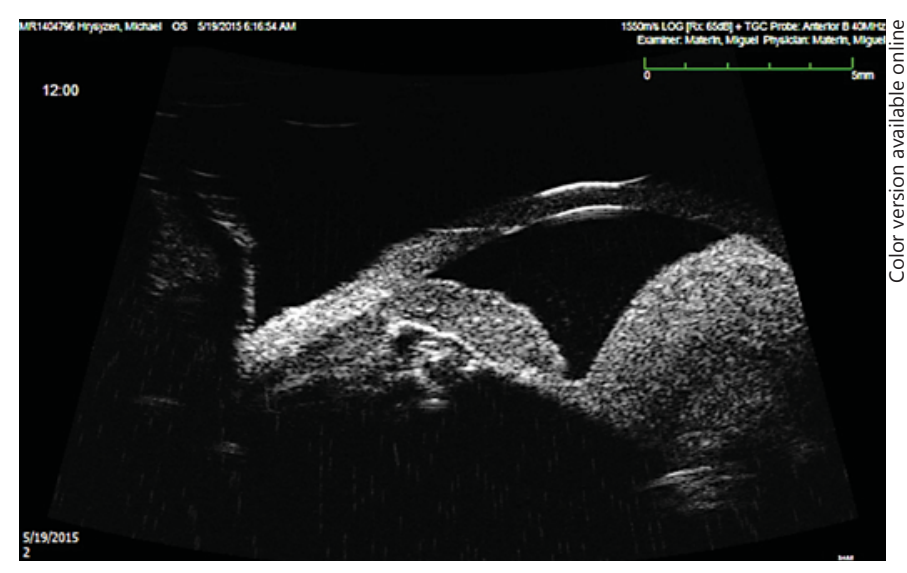

Fig. 2. Anterior segment ultrasound biomicroscopy of the left eye at presentation reveals an iris mass.

hyphemas and elevated ocular tensions leading to glaucoma in the left eye that was presumed to be related to his cataract surgery.

Of relevance, the patient had a history of mantle cell lymphoma (MCL) that was diagnosed 13 years prior to referral by biopsy of a lymph node in the groin region. He was treated with rituximab, cyclophosphamide, doxorubicin, and vincristine. He was considered in remission until about 4 years before referral when a bone marrow biopsy suggested recurrence of MCL. This was then treated with bendamustine and ritixumab. A bone marrow biopsy 2 years later was negative for recurrence.

On presentation to ophthalmic oncology, his vision was $20 / 50$ in the right eye and light perception in the left eye. His intraocular pressures were $15 \mathrm{~mm} \mathrm{Hg}$ in the right eye and $22 \mathrm{~mm} \mathrm{Hg}$ in the left eye, and there was a relative afferent pupillary defect in the left eye. The left eye exam was notable for dilated and tortuous episcleral

Iris Lymphoma Masquerading as UGH Syndrome vessels, a layered hyphema occupying $30 \%$ of the anterior chamber volume, and circumferential engorgement of the iris that narrowed the anterior chamber depth (fig. 1). Anterior chamber red blood cells and abnormal iris tissue precluded a view to the posterior segments. Anterior segment ultrasound biomicroscopy demonstrated an iris mass that appeared to spare the ciliary body (fig. 2); posterior ultrasonography showed choroidal detachment. His right eye exam had no features suggestive of lymphomatous involvement.

The differential diagnosis at the time included secondary lymphomatous involvement of the iris and amelanotic melanoma. Given the poor visual potential of the left eye, primary enucleation was offered as a diagnostic and therapeutic option. However, the patient declined this option. A punch biopsy was attempted in the operating room but was unsuccessful due to hard tissue density. As a result, intraocular scissors were used to obtain an incisional tissue biopsy instead. Histopathologic examination of the tissue showed a monotonous population of CD20+ lymphocytes compatible with a B-cell lymphoma that was unable to be further specified due to specimen size (fig. 3). One week later, a positron emission tomographic scan demonstrated lymphomatous recurrence in his neck and chest. Medical oncology has been treating the patient with ibrutinib. In the most recent follow-up visit with ophthalmology 4 months after referral, the iris appears slightly regressed (fig. 4). The patient's left eye vision remains light perception only.

\section{Discussion}

NHL consists of a vast array of neoplastic B-cell lymphomas that are subclassified based on morphologic, immunohistochemical, flow cytometric, and genetic features. While some NHLs are indolent, such as chronic lymphocyte leukemia, others behave more aggressively and require more intensive therapy [8]. MCL generally presents at late stage, followed by an aggressive clinical course. However, there is a subset of patients with indolent MCL. Morphologically, in its classic form, MCL consists of small to intermediate-sized lymphocytes with irregular, cleaved nuclear contours, condensed chromatin, indistinct nucleoli and a small amount of cytoplasm (fig. 3a). Since the neoplastic lymphoid cells are derived from B cells, immunohistochemistry (IHC) is positive for $\mathrm{CD} 20$ (fig. 3b); coexpression of CD5 is also characteristic of MCL. The defining genetic event of MCL is translocation at $\mathrm{t}(11 ; 14)(\mathrm{q} 13 ; \mathrm{q} 32)$ resulting in rearrangement of the BCL1 gene and overexpression of cyclin D1. The translocation is assessed directly by cytogenetic analysis or more commonly by IHC. In our case, cyclin D1 was identified only in a small subset of cells (fig. 3c). CD10 is characteristically absent from MCL (fig. 3d) and helps differentiate it from follicular lymphoma, in which it is usually expressed. 


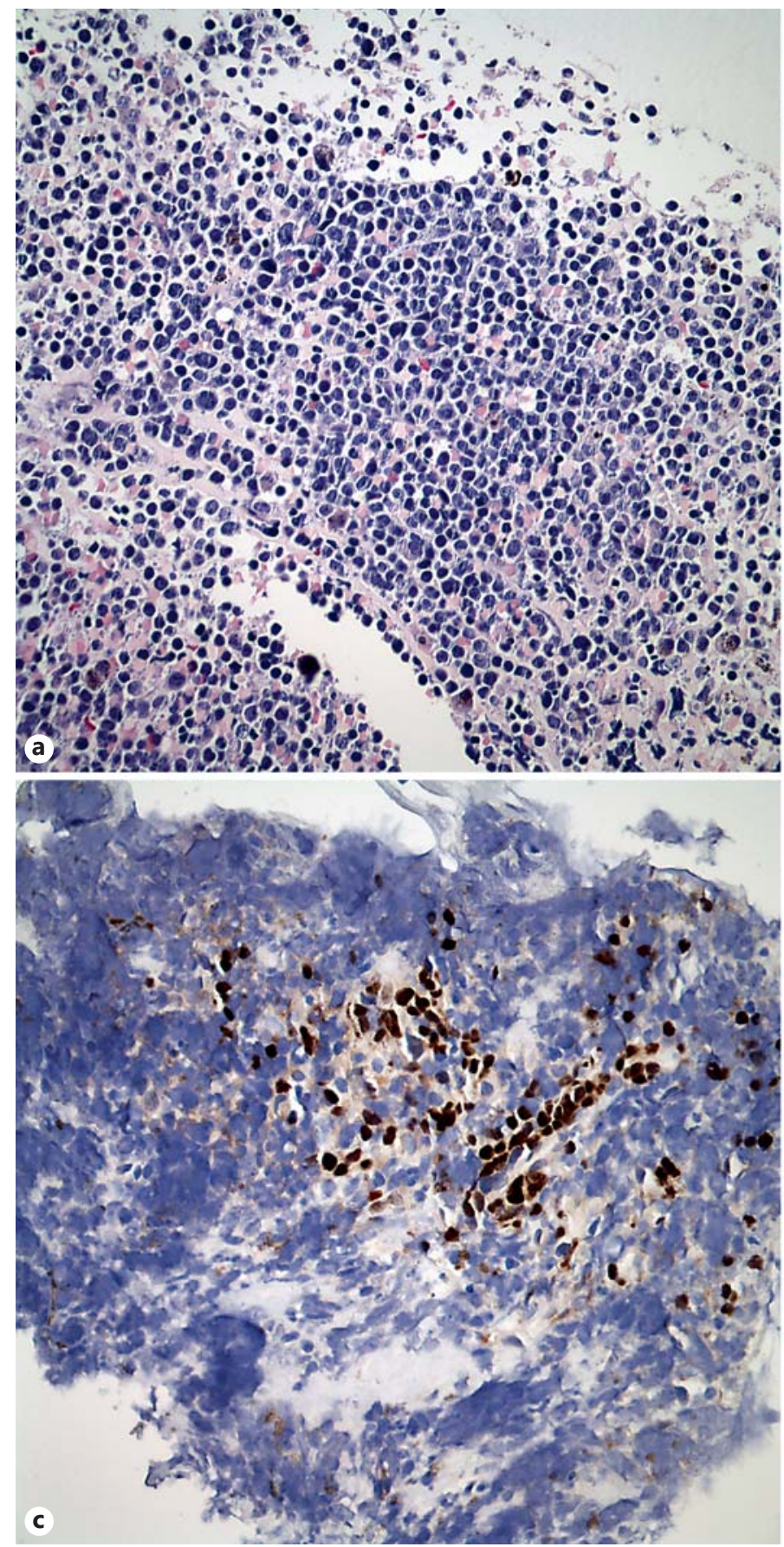

Fig. 3. Iris lymphoma. a Scattered intermediate-sized lymphocytes with irregular nuclear contours, open chromatin, variably distinct nucleoli and a small amount of cytoplasm are noted. Focal singlecell necrosis is present and mitotic figures are easily identified. HE.

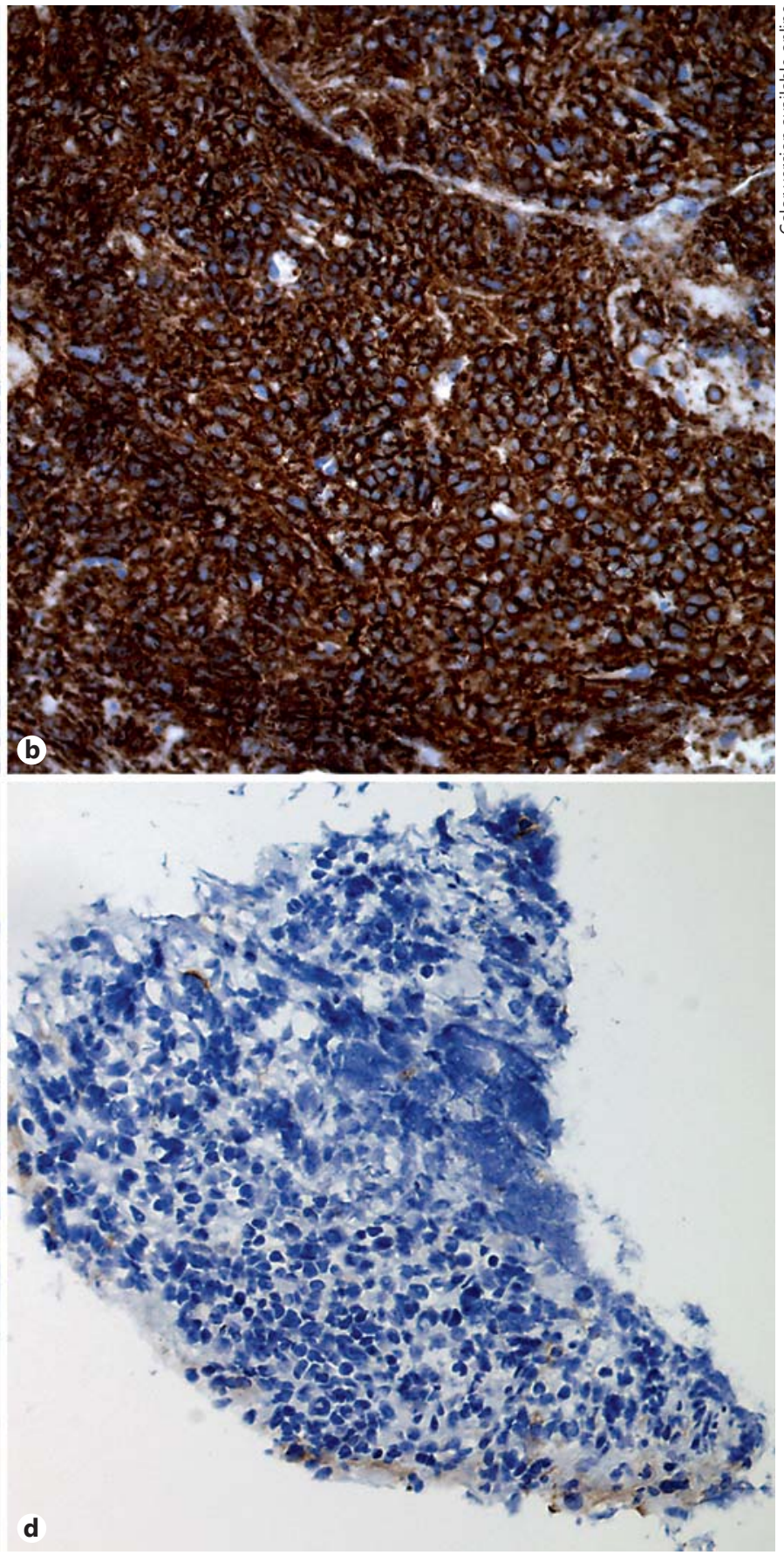

$\times 40$. b CD20 immunohistochemistry is strongly and diffusely positive. IHC. $\times 40$. Cells show scattered positivity for cyclin D1 (c) and are negative for CD10 (d). IHC. $\times 40(\mathbf{c}), \times 20$ (d). 


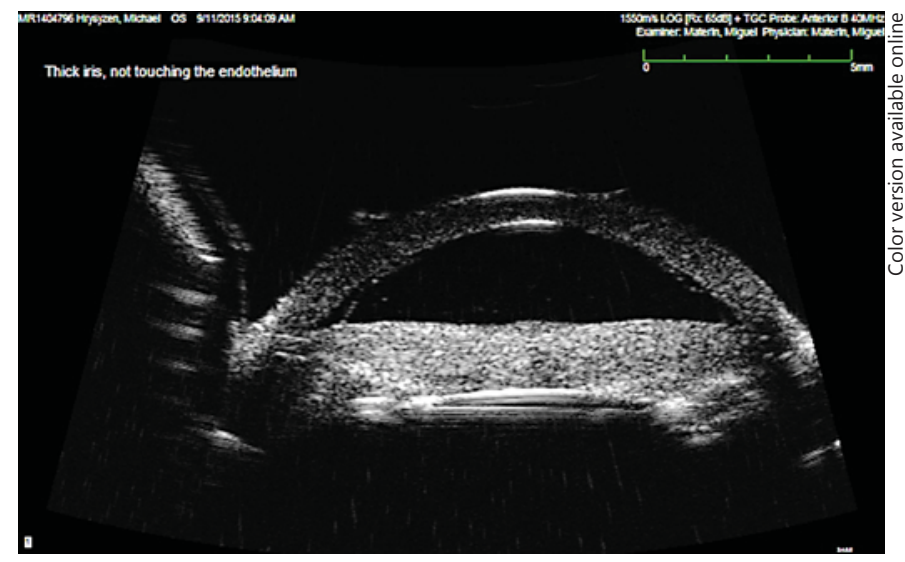

Fig. 4. Photograph of left eye 4 months after referral shows a slightly regressed iris mass.

The biopsy from this patient showed a non-Hodgkin Bcell lymphoma with aggressive cytomorphologic features including open chromatin, variably distinct nucleoli, easily identified mitotic figures, and single-cell necrosis. Since it did not meet the morphologic criteria for a large cell lymphoma, MCL was highly suspected even in the absence of clinical history at the time of pathologic evaluation. However, due to only subset staining of cyclin D1 and limited tissue for other helpful markers such CD5 or SOX11, definitive lymphoma subclassification could not be rendered on this biopsy alone. While a punch biopsy often provides sufficient tissue for IHC analysis, the high tissue density encountered precluded this sampling approach. Needle biopsies of iris tissue are generally discouraged.
Lymphomatous involvement of the iris is a rare entity that can be difficult to distinguish from more common causes of anterior uveitis. However, an accurate and timely diagnosis is essential given that the condition poses a threat to life. In one case series, it was noted that half of all eyes with iris lymphoma presented with a hyphema [6]. It was suggested that the presence of a hyphema may be helpful for distinguishing cases of iris lymphoma from other causes of anterior uveitis. The differential diagnosis of anterior uveitis becomes greatly narrowed in the presence of a hyphema, which includes uveitides in association with various seronegative spondyloarthropathies and herpes simplex virus [9]. The case presented herein was particularly difficult to diagnose early on as the onset of the ocular symptoms closely followed cataract extraction. In this context, a diagnosis of UGH syndrome seemed fitting initially, and as a result, an accurate diagnosis was delayed.

\section{Statement of Ethics}

Consent was obtained from the patient as appropriate. The case reported herein was exempt from our human research protection office.

\section{Disclosure Statement}

Financial support: this work was funded in part by an unrestricted departmental grant from Research to Prevent Blindness (RPB), Inc. None of the other authors of the manuscript have conflicts of interests to disclose.

\section{References}

1 Boffetta PI: Epidemiology of adult nonHodgkin lymphoma. Ann of Oncol 2011;22: $27-31$.

2 Domingo RE, Manganip LE, Castro RM: Tumors of the eye and ocular adnexa at the Philippine Eye Research Institute: a 10-year review. Clin Ophthalmol 2015;9:1239-1247.

3 Coupland SE, Damato B: Lymphomas involving the eye and the ocular adnexa. Curr Opin Ophthalmol 2006;17:523-531.
4 Yamada K, Hirata A, Kimura A, Tanihara H: A case of primary B-cell type non-Hodgkin lymphoma originating in the iris. Am J Ophthalmol 2003;136:380-382.

5 Economou MA, Kopp ED, All-Ericsson C, Seregard S: Mantle cell lymphoma of the iris. Acta Ophthalmol Scand 2007;85:341-343.

6 Mashayekhi A, Shields CL, Shields JA: Iris involvement by lymphoma: a review of 13 cases. Clin Experiment Ophthalmol 2013;41:19-26.

7 Van Liefferinge T, Van Oye R, Kestelyn P: Uveitis-glaucoma-hyphema syndrome: a late complication of posterior chamber lenses. Bull Soc Belge Ophtalmol 1994;252:61-65; discussion 66.
8 Campo E, Swerdlow SH, Harris NL, Pileri S, Stein H, Jaffe ES: The 2008 WHO classification of lymphoid neoplasms and beyond: evolving concepts and practical applications. Blood 2011;117:5019-5032.

9 Fong DS, Raizman MB: Spontaneous hyphema associated with anterior uveitis. Br J Ophthalmol 1993;77:635-638. 